\title{
Clinical and Laboratory Findings in COVID-19 Patients: Comparison of RT-PCR Positive and Negative Cases
}

\author{
Farnaz karimi ${ }^{1}$, Amir Vaezi $^{1}$, Mostafa Qorbani ${ }^{1}$, Fatemeh Moghadasi ${ }^{1}$, Saeed Hasani \\ Gelsefid $^{1}$, Arman Maghoul ${ }^{1}$, Neda Mahmoodi ${ }^{1}$, Zahra Eskandari ${ }^{2}$, Hossein Gholami ${ }^{1}$, \\ Zakiye Mokhames ${ }^{1}$, and Mahshid Saleh ${ }^{3}$ \\ ${ }^{1}$ Alborz University of Medical Sciences \\ ${ }^{2}$ Shahid Beheshti University of Medical Sciences \\ ${ }^{3}$ Affiliation not available
}

June 24, 2020

\begin{abstract}
The novel coronavirus disease 2019 (COVID-19) has turned into a global public health emergency after the first patients were detected in Wuhan, China, in December 2019. Methods This case-control study was conducted in two hospitals of Alborz Province in Iran. All symptomatic adults hospitalized as COVID-19 patients with compatible CT scan findings and available RT-PCR results were recruited in this study. The patients were categorized in positive and negative RT-PCR groups and evaluated for symptoms, initial vital signs, comorbidity, clinical and laboratory findings and the results were assessed by SPSS software. Results Between March 5 to April 5, 2020, 164 symptomatic COVID-19 patients were studied. In total, there were 111 RT-PCR positive (67.6\%) and 53 RT-PCR negative patients (32.4\%). In terms of statistics, the frequency of symptoms revealed no difference, except for cough (P.V:0.008), dizziness (P.V: 0.048) and weakness (P.V:0.022). Among initial vital signs, PR (P.V:0.041) and O2 Saturation (P.V: 0.014) were statistically different between the two groups. Evaluation of comorbidities revealed no difference except for hyperlipidemia (P.V:0.024). In comparison of laboratory findings, only WBC count (P.V: 0.001), lymphocyte count (P.V: 0.001) and Hb (P.V:0.008) were statistically different between the two groups. Conclusion In case of negative RT-PCR result, it is necessary to take a logical approach, and we recommended that the physician decide according to clinical manifestations, laboratory findings, and positive CT result
\end{abstract}

\section{Introduction:}

At the end of 2019, a novel coronavirus was identified as the cause of a cluster of pneumonia cases in Wuhan, a city in the Hubei Province of China. It rapidly spread, resulting in an epidemic throughout China, followed by an increasing number of cases in other countries throughout the world. (1). Following the detection and announcement of the first cases of COVID-19 on February 20, the infection rate and death toll from the disease has been on the rise (2). The responsible causative agent, namely SARS-CoV-2, is an enveloped RNA virus of Coronaviridae family. The transmission of COVID19 occurs via respiratory droplets or contaminated surfaces (3). The symptoms of COVID-19 are non-specific, which range from asymptomatic to severe pneumonia and can lead to death (4). The majority of patients present with mild respiratory tract infection, mostly commonly fever (82\%) and cough (81\%). Severe pneumonia and acute respiratory distress syndrome (ARDS) have been reported in $14 \%$ of cases with overall mortality rate of $2 \%(5)$. Nevertheless, these figures are rising concomitant with the expansion of pandemic depending on the country involved (6). SARS-CoV infection in humans leads to an acute respiratory illness varying from mild febrile disease to ALI and in some cases ARDS and death $(7,8)$. Considering the high infectivity of COVID-19, rapid and precise 
diagnostic methods are urgently required to detect, isolate and treat the patients as soon as possible, which might decrease mortality rates and the risk of community contamination (9). Computed tomographic (CT) imaging is extensively applied for the early diagnosis of COVID-19, but chest CT may not distinguish this disease from other viral pneumonias $(10,11)$. Nucleic acid assay, gene sequencing, and serology tests (IgM and $\operatorname{IgG}$ ) from throat swabs or blood samples have been developed to confirm the diagnosis of COVID19 (12). However, clinicians from Wuhan have addressed the issue of high false negative rates of PCR or antibody detection (13). However, rRT-PCR results often require 5 to 6 hours to be prepared, while CT results can be obtained much faster (9). Chunqin Long et al. revealed that the sensitivity of CT examination was $97.2 \%$ at presentation, whereas the sensitivity of first round rRT-PCR was $84.6 \%$ (9). This difference may be a function of sample collection because pharyngeal and nasal sampling are more straightforward collection methods, while lower respiratory tract sampling is relatively difficult to perform and bears the risk of infection for susceptible medical staff (14). The sensitivity of rRT-PCR kit can also give rise to false negative results (9).

Simultaneous use of medical history, clinical manifestations, chest CT, and viral test has been found to present high sensitivity $(92-97 \%)(15,16)$.

A major challenge for the restriction of SARS-CoV-2 spread is that pre-symptomatic people are infectious for others (17). Recent reports indicated that patients can be infectious 1-3 days before the onset of symptoms and that up to $40-50 \%$ of cases may be attributable to transmission from asymptomatic or presymptomatic individuals $(18,19)$. Patients have high nasopharyngeal loads of virus just before or soon after the onset of symptoms, which subsequently fall over the course of approximately one week (20). Patients with severe disease can release the virus for longer periods, although the shedding duration of infectious virus is not precisely known (21). Evaluation and management of COVID-19 is determined by the severity of the illness. According to initial data taken from China, $81 \%$ of people with COVID-19 had mild or moderate disease (including people without pneumonia and those with mild pneumonia), $14 \%$ had severe disease, and $5 \%$ showed critical illness (22).

According to the guidelines of World Health Organization (WHO), the risk of COVID-19 virus infection is not ruled out if one or more negative PCR tests are observed. Factors such as low sample quality (lack of sufficient DNA in the sample), inappropriate time of sampling (delayed or early sampling), improper storage and transportation of the sample, and inherent technical reasons for testing including virus mutations or PCR inhibitions (such as improper swap use, etc.,) can affect PCR testing and lead to false negative responses (23).

Due to the rapid spread of COVID19 disease in Iran, increasing number of patients in a short period of time, lack of cooperation of some patients in the sampling process, limited access to RT-PCR testing, assuming PCR as a time consuming test and inability to repeat negative tests, many suspected patients with COVID19 were hospitalized in medical centers based on clinical symptoms, vital signs, laboratory data and CT-Scan findings.

Due to limitation of available hospital beds, some of the hospitalized patients showing minor clinical symptoms, mild CT-Scan changes and improved vital signs who had negative PCR results were discharged by physician's clinical judgement within a few days of admission. There were several reports of exacerbated symptoms and re-hospitalization of patients in severe conditions .Meanwhile, the positive or negative PCR results have at times led the physicians into misinterpretation of patient's clinical conditions. The decision to continue treatment or discharge the patient from the hospital in those with mild clinical symptoms, negative PCR test and positive CT is an important question for physicians.

Accordingly, the goal of this study is to investigate the importance of clinical symptoms, initial vital signs, laboratory findings and chest CT-Scan findings among patients with negative and positive PCR results.

\section{Methods \& Materials:}

This research was a case-control study that was performed in two hospitals in Alborz Province of Iran. 
This study was approved by the ethics committee of Alborz University of Medical Sciences with ethics code IR.ABZUMS.REC 1398.267. Informed consent was taken from subjects and the inclusion criteria were as follows: all patients with related signs and symptoms of COVID-19 and compatible CT findings requiring hospitalization according to National Guideline items $\left(\mathrm{RR}>33\right.$ or $\mathrm{O}_{2}$ saturation $\left.<93\right)$ or physician's judgement. Exclusion criteria were inconclusive PCR results, causes of pneumonia other than COVID-19, unclear or incomplete recorded history of patients. Data were collected from the patient's medical findings and self-reports in determined categories.

\section{Patients and Study design:}

A total of 164 patients participated in this study from March 5 to April 5, 2020. We divided the patients into two groups: 111 PCR positive $(67.6 \%)$ and 53 PCR negative patients (32.4\%). CT scans were performed before or at the time of admission for all symptomatic patients and all CT scan results were compatible with COVID -19. The patients were evaluated for symptoms, initial vital signs, comorbidity, clinical and laboratory findings (CBC, biochemistry parameters, inflammation indices) and date of symptoms' onset before admission. Real-time reverse transcriptase polymerase chain reaction (RT-PCR) was performed on oropharyngeal specimens. In this study, all samples were tested within 48 hours after hospitalization and sampling was done using a standard protocol by trained individuals.

\section{CT scan:}

Typical and atypical chest CT findings were documented based on CT features defined for COVD-19. Radpour et al. designed a low-dose high resolution computed tomography (HRCT) protocol for the Iranian Society of Radiology to assess patients bearing a high chance of COVID-19 infection. The recommended parameters to diminish the radiation dose were as follows: Kvp: 100-120, mAs: 50-100, pitch: 0.8-1.5, thickness: 1-3 mm, which were used in the present research (24).

\section{RT-PCR:}

All samples were subjected to RNA extraction with Qiagen Viral Nucleic Acid Kit (QIAcub HT) according to the manufacturer's instructions. RT-PCR was used to detect the presence of SARS-CoV2 using the kits (Molbiol, Germany) provided by WHO targeting the E region for screening and RNA dependent RNA polymerase for confirmation. Invitrogen Superscript III One-Step RT-PCR System with Platinum Taq DNA Polymerase was used for PCR. For each reaction, $15 \mu \mathrm{l}$ reaction mix, $1 \mu \mathrm{l}$ RT enzyme, $0.5 \mu \mathrm{l}$ primer, probe mix and $3.5 \mu \mathrm{l} \mathrm{PCR}$ grade water were added to $5 \mu \mathrm{l} \mathrm{RNA}$ template. Cycling conditions for amplification of $\mathrm{E}$ and RdRP genes were $50^{\circ} \mathrm{C}$ for $30 \mathrm{~min}, 95^{\circ} \mathrm{C}$ for $2 \mathrm{~min}$, followed by 45 cycles of $95^{\circ} \mathrm{C}$ for 10 sec and $60^{\circ} \mathrm{C}$ for 30 sec. A cycle threshold value of $<36 \mathrm{Ct}$ was defined as a positive test result.

\section{Data analysis:}

We divided all suspected patients into the control and case groups based on RT-PCR test results. The case group included the suspected patients with a positive RT-PCR result, and the control group consisted of patients with a negative RT-PCR result. Continuous and categorical variables were compared using t-test, Mann-Whitney U test and Chi square test, cross tabulation between RT-PCR positive and negative groups, respectively. The data were expressed as mean \pm standard deviation (SD) and median, interquartile range (IQR) in continuous variables and frequency (percentage) in categorical data. The SPSS software version 22.0 was used for statistical analysis and $\mathrm{P}<0.05$ was considered statistically significant.

\section{Results:}

\section{Clinical characteristics of the patients:}

164 subjects (44.5\% female \& $55.5 \%$ male) with a mean age of 54 (range: $24-89)$ years were studied.

The result of RT-PCR for COVID-19 was positive in 111 patients (67.6\%) and 53 patients (32.4\%) showed negative PCR results. Positive and negative RT-PCR results are shown based on gender $(\mathrm{P} . \mathrm{V}=0.384)$ and age $(\mathrm{P} . \mathrm{V}=0.075)$ distribution in Table 1. 
Comparing the symptoms in positive and negative RT-PCR groups showed no significant difference except for dizziness $(\mathrm{p}=0.048)$, cough $(\mathrm{p}=0.008)$ and weakness $(\mathrm{p}=0.022)$, which were slightly more frequent in positive RT-PCR group than the negative RT-PCR group.

Cough $(31,58.5 \%)$, dyspnea $(93,57.1 \%)$, fever $(60,54.1 \%)$ and weakness $(61,55.0 \%)$ were the most common symptoms in positive RT-PCR group. In negative RT-PCR group, cough (87, 78.4\%), dyspnea (29, 54.7\%), fever $(23,43.4 \%)$ and muscular pain $(23,43.4)$ were the most prevalent symptoms.

Statistical analysis of initial vital signs showed that RR, Temp, SBP, and DBP were similar between positive and negative RT-PCR groups, while pulse rate $\mathrm{PR}(\mathrm{HR})(\mathrm{p}<0.041)$ and $\mathrm{O}_{2}$ saturation $(\mathrm{SO} 2)(\mathrm{p}<0.014)$ were significantly different in the two groups, which are shown in Table 1.

In positive RT-PCR group, the average (mean +-SD) of PR (HR) and $\mathrm{SO}_{2}$ were 99.4 (17.2) and 91.8 (5.5), respectively, which were 98.6 (23.0) and 89.5 (8.0) in negative RT-PCR group

According to the data, the average PR was slightly higher in positive RT-PCR group and the average of $\mathrm{SO}_{2}$ in the negative RT-PCR group was slightly lower.

\title{
Clinical findings:
}

With respect to comorbidities, the two groups had significant similarities, and only hyperlipidemia was significantly higher in positive RT-PCR group $(10,9.0 \%)(\mathrm{p}=0.024)$. The most common comorbidities in positive RT-PCR group were hypertension and diabetes $(28,25.2 \%)$ and $(27,24.3 \%)$, respectively. Also, in negative RT-PCR group, hypertension $(11,20.8 \%)$ and diabetes $(12,22.6 \%)$ were the most common comorbidities (Table 1).

We also analyzed the appearance date of symptoms before admission and found no significant difference between groups (Table 2).

\section{Laboratory parameters:}

Table 2 compares the laboratory parameters of patients with positive and negative RT-PCR results. There were many similarities in laboratory findings between positive and negative RT-PCR groups, among which WBC $(\mathrm{p}=0.001)$, lymphocyte count $(\mathrm{p}=0.001)$ and $\mathrm{Hb}(\mathrm{p}=0.008)$ were statistically significant.

In positive RT-PCR group, white blood cell median (IQR) was 5.4 (4.2-6.8), lymphocyte count median (IQR) was 1.1 (0.879-1.4) and Hb mean was 13.7 (1.6). In negative RT-PCR group, the following values were as follows: white blood cell median (IQR) 5.4 (4.2-6.8), lymphocyte count median (IQR) 1.1(0.8-1.4) and Hb (mean +-SD) 13.7 (1.6).

Clinical characteristics and initial vitals of the patients in RT-PCR positive and negative groups(Table 1)

\author{
Variables \\ Gender NO\% \\ Female \\ Male \\ Initial vital sign. Mean(SD) \\ Age \\ $\mathrm{RR}$ \\ Temp Temp $>37.8$ \\ PR \\ Sys BP, mm Hg \\ Dias BP, mm Hg \\ O2 Sat O2 Sat $<90 \%$ \\ Symptoms.NO(\%) \\ Cough \\ Dyspnea
}


Clinical characteristics and initial vitals of the patients in RT-PCR positive and negative groups(Table 1)

Sore throat

Fever

Chills

Headache

Dizziness

Weakness

Muscular pain

Diarrhea

Abdominal pain

Anorexia

Nausea

Vomiting

Comorbidity.NO(\%)

Diabetes

Hyperlipidemia

Hypertension

CHD

CKD

Asthma

COPD

Cirrhosis

Autoimmune disease

History of malignancy

Recent chemoradiotherapy

Current steroid use

Immunosuppressant drug use

$\mathrm{RR}=$ Respiratory rate. Temp $=$ Temperature. $\mathrm{PR}=$ Pulse rate. Sys $\mathrm{BP}=$ Systolic blood pressure. Dias $\mathrm{BP}=\mathrm{Diastolic}$ blooc

Laboratory findings of patients infected with 2019-nCoV on admission to hospital in PCR Positive and PC]

Variable( $\mathrm{n} / \mathrm{N}$ )

$\mathrm{Hb} \mathrm{mg} / \mathrm{dl}$

WBC count, $\times 109 / \mathrm{L}$

Lymph count, $\times 109 /$ L Lymph count $<1.1 \times 109 / \mathrm{L}$

Platelet count, $\times 109 / \mathrm{L}$

$\operatorname{ESR}(124 / 164)$

CRP mg/dl (159/164) CRP $>6 \mathrm{mg} / \mathrm{dl}$

LDH, U/L (104/164) LDH $>245$

AST, U/L( 80/164)

ALT, U/L (80/164)

CPK U/L(23/164)

Sodium mmol/L (130/164)

Potassium mmol/L (130/164)

Magnesium mmol/L (60/164)

BUN mg/dl(158/164)

Cr mmol/L (158/164)

Date of symptoms before admission

This data is Mean(SD) Other data are Median (IQR), or n/N (\%) Hb= Hemoglobin. WBC= White blood cell. Lymph= L 


\section{Discussion:}

The global outbreak of novel coronavirus 2019 (COVID-19) has been a matter of international concern due to the fast spread of the disease (25). During the initial phase of COVID-19 outbreak, the diagnosis of the disease was complicated due to the diversity of symptoms and imaging results as well as the severity of disease at the time of presentation (26). Therefore, it is very essential to precisely diagnose the patients suspected with COVID-19 infection for appropriate isolation or treatment. Currently, the RT-PCR amplification of viral DNA is considered as the "gold standard". However, initial RT-PCR is not always positive in a patients with COVID-19 infection $(16,27)$. In this case, chest CT images could play an important role to detect the lesions of pulmonary parenchyma in the patients suspected with COVID-19 infection. Nevertheless, it does not mean that the abnormalities of CT images could be observed in COVID-19 infection while the initial RT-PCR is positive or negative $(16,27,28)$.

Previous studies have suggested that a false-negative rRT-PCR result may occur in some COVID-19 patients $(29,30)$. False-negative results may be a function of various factors such as human errors when following the diagnostic kit protocol, the sensitivity of reagents, the site and method of specimen sampling and collection times (31).

In Yang et al. study, the total positive rate of RT-PCR for throat swab samples was reported to be about $30-60 \%$ at initial presentation despite limitations of sample collection, transportation, and kit performance. In this study, all patients were evaluated for clinical manifestations and radiological examination (32). One of the studies in Wuhan revealed that a considerable ratio of COVID-19 patients may have had an initial negative rRT-PCR result and that the primarily positive patients had a higher tendency to progress to severe cases. In this study, it was stated that patients with negative rRT-PCR who presented with typical clinical manifestations should not be ignored and suggested that PCR should be repeated (33).

In our study, we also examined the date of symptoms' onset before admission to the hospital and no significant differences were observed between PCR positive and PCR negative groups. Yang et al. revealed that the sputum sample collected during 8-14 days showed a higher positive qPCR rate than the nasal and throat swabs samples in both severe and mild cases. The positive qPCR rate of throat samples decreased a few days after the onset of symptoms to hospitalization and performing PCR tests. The likelihood of positive throat samples test and symptoms decreased after 15 days (32).

In our study, patients were hospitalized based on clinical manifestations, the results of laboratory tests and positive CT scan corresponding to COVID-19, and RT-PCR test result were ready after 48 hours. We performed RT-PCR on oropharyngeal specimens. In this research, we found $67.6 \%$ PCR positive results, a percentage that may be due to the same test conditions, including the operator performing the test, sampling method, diagnostic kit, etc. for all samples. We detected a slight difference between positive and negative RT-PCR patients in terms of clinical and laboratory findings, initial vital signs and comorbidity. Therefore, patients with negative RT-PCR should not be discharged from hospital, especially when presenting similar clinical manifestations to positive RT-PCR patients. It can be strange that those negative RT-PCR results could be problematic.

This study has a number of limitations. First, it was not possible to repeat negative RT-PCR tests due to shortcomings such as the lack of laboratory testing capacity, insufficient staff and limited number of diagnostic kits. Second, RT- PCR tests were performed only on hospitalized patients and did not assess COVID-19 suspects who had been recommended home quarantine and rest. Third, the physicians judged the patient's hospitalization requirement based on clinical symptoms and CT scan of lungs because it was not possible to perform a rapid RT-PCR test at first. Therefore, we had no patients with negative CT scan and positive RT-PCR. Fourth, incomplete medical records of a few patients due to the high number of patients' admission to the hospital emergency ward, insufficient number of physicians and nurses to complete the history as well as the patients' inability to express their history were another limitation of the present research.

\section{Conclusion}


Finally, we conclude that the decision on COVID-19 patients should not exclusively depend on RT-PCR positivity during the time of the pandemic. Clinical manifestations, laboratory findings and positive CT results play a critical role in clinician's decision, especially in countries with a high prevalence of COVID19 with lower medical facilities. The patients would be isolated from other healthy individuals and not discharged from the hospital until they have fully recovered. We suggest that it would be better to investigate a large number of people to obtain more accurate results. This study compared clinical characteristics, initial vital signs and laboratory data of early stage of COVID-19 in PCR positive and negative groups in Iran, which has certain value for future control and research. Follow-up of the patients and outcome evaluation between RT-PCR positive and negative groups could be the subjects for further research.

\section{Ethics approval and consent to participate:}

Written informed consent was obtained from each patient or the patient's legally authorized surrogate prior to conduct of study-specific procedures.

\section{Competing interests:}

The authors declare that they have no competing interests.

\section{Funding}

This work was supported by the Alborz University of Medical Sciences.

Funding for this study (ethics code IR.ABZUMS.REC 1398.267) was provided by Alborz university of Medical Sciences.

\section{Authors'Contributions}

FK and AV contributed to the concept of the review. FK, AV, MS, FM, SH, AM, MQ, ZM, HGH and NM, ZE were responsible for the reference selection and writing of the manuscript. FK, AV and MS contributed to the critical review of the manuscript. All authors read and approved the final manuscript.

\section{Acknowledgements}

The authors thank Dr.Mohammad Noori Sepehr for his contributions to the study.

\section{Author information}

\section{Affiliations}

Farnaz Karimi ${ }^{1}$, Amir Abbas Vaezi ${ }^{2}$, Mostafa Qorbani ${ }^{3,4}$, Fatemeh Moghadasi ${ }^{5}$, Saeed Hasani gelsefid ${ }^{6}$, Arman Maghoul ${ }^{7}$, Neda Mahmoodi ${ }^{8}$, Zahra Eskandari ${ }^{9}$, Hossein Gholami ${ }^{10}$, Zakiye mokhames ${ }^{11}$, Mahshid Saleh ${ }^{12}{ }^{*}$.

1. Department of pathology, Alborz University of Medical science, karaj, Iran.

2. Department of internal medicine, Alborz University of Medical Science ,karaj ,Iran

3. Non-Communicable Diseases Research Center, Alborz University of Medical Sciences, Karaj, Iran.

4. Chronic Diseases Research Center, Tehran University of Medical Sciences Endocrinology and Metabolism Research Institute, Tehran, Iran.

5. Faculty of medicine, Alborz University of Medical Science karaj, Iran.

6. Faculty of medicine, Alborz University of Medical Science karaj, Iran.

7. Faculty of medicine, Alborz University of Medical Science karaj, Iran.

8. Imam Jafar sadegh hospital, Alborz University of Medical Science karaj, Iran.

9. Department of Health in emergencies and disasters Shahid Beheshti University of Medical Science, Tehran, Iran.

10. Imam Ali hospital, Alborz University of Medical Science karaj, Iran.

11. Department of molecular diagnostics, Alborz University of medical Sciences Karaj, Iran.

12. Tissue Engineering and Applied Cell Sciences, Tehran University of Medical Science, Tehran,Iran. 


\section{Corresponding author:}

Mahshid Saleh, Department of Tissue Engineering and Applied Cell Sciences,Faculty of Advanced Technologies in Medicine,Tehran University of Medical Science,Tehran, IRAN .Tel: (+989127249532),

Email:m_saleh@razi.tums.ac.ir.

\section{References:}

1. McIntosh K, Hirsch MS, Bloom A. Coronavirus disease 2019 (COVID-19). 2020] Disponível em: https://www uptodate com/contents/coronavirus-disease-2019-covid-19-epidemiology-virology-clinicalfeatures-diagnosis-and-prevention Acesso em. 2020;30.

2. Zandifar A, Badrfam R. Fighting COVID-19 in Iran; economic challenges ahead. Archives of Iranian medicine. 2020;23(4):284.

3. Zhu N, Zhang D, Wang W, Li X, Yang B, Song J, et al. A novel coronavirus from patients with pneumonia in China, 2019. New England Journal of Medicine. 2020.

4. Guan W-j, Ni Z-y, Hu Y, Liang W-h, Ou C-q, He J-x, et al. Clinical Characteristics of Coronavirus Disease 2019 in China. New England Journal of Medicine. 2020;382(18):1708-20.

5. Sheahan TP, Sims AC, Leist SR, Schäfer A, Won J, Brown AJ, et al. Comparative therapeutic efficacy of remdesivir and combination lopinavir, ritonavir, and interferon beta against MERS-CoV. Nature Communications. 2020;11(1):1-14.

6. Khoury M, Cuenca J, Cruz FF, Figueroa FE, Rocco PRM, Weiss DJ. Current Status of Cell-Based Therapies for Respiratory Virus Infections: Applicability to COVID-19. European Respiratory Journal. 2020:2000858.

7. Peiris J, Guan Y, Yuen K. Severe acute respiratory syndrome. Nature medicine. 2004;10(12):S88-S97.

8. Peiris J, Lai S, Poon L, Guan Y, Yam L, Lim W, et al. Coronavirus as a possible cause of severe acute respiratory syndrome. The Lancet. 2003;361(9366):1319-25.

9. Long C, Xu H, Shen Q, Zhang X, Fan B, Wang C, et al. Diagnosis of the Coronavirus disease (COVID-19): rRT-PCR or CT? European journal of radiology. 2020:108961.

10. Kanne JP. Chest CT findings in 2019 novel coronavirus (2019-nCoV) infections from Wuhan, China: key points for the radiologist. Radiological Society of North America; 2020.

11. Pan Y, Guan H. Imaging changes in patients with 2019-nCov. Springer; 2020.

12. Xu G, Yang Y, Du Y, Peng F, Hu P, Wang R, et al. Clinical Pathway for Early Diagnosis of COVID-19: Updates from Experience to Evidence-Based Practice. Clinical Reviews in Allergy \& Immunology. 2020:1-12.

13. Pan F, Ye T, Sun P, Gui S, Liang B, Li L, et al. Time course of lung changes on chest CT during recovery from 2019 novel coronavirus (COVID-19) pneumonia. Radiology. 2020:200370.

14. Tian H. 2019-nCoV: new challenges from coronavirus. Zhonghua yu fang yi xue za zhi [Chinese journal of preventive medicine]. 2020;54:E001-E.

15. Jiang G, Ren X, Liu Y, Chen H, Liu W, Guo Z, et al. Application and optimization of RT-PCR in diagnosis of SARS-CoV-2 infection. medRxiv. 2020.

16. Ai T, Yang Z, Hou H, Zhan C, Chen C, Lv W, et al. Correlation of chest CT and RT-PCR testing in coronavirus disease 2019 (COVID-19) in China: a report of 1014 cases. Radiology. 2020:200642.

17. Kimball A, Hatfield KM, Arons M, James A, Taylor J, Spicer K, et al. Asymptomatic and presymptomatic SARS-CoV-2 infections in residents of a long-term care skilled nursing facility-King County, Washington, March 2020. Morbidity and Mortality Weekly Report. 2020;69(13):377. 
18. Wei WE, Li Z, Chiew CJ, Yong SE, Toh MP, Lee VJ. Presymptomatic Transmission of SARS-CoV-2Singapore, January 23-March 16, 2020. Morbidity and Mortality Weekly Report. 2020;69(14):411.

19. He X, Lau EH, Wu P, Deng X, Wang J, Hao X, et al. Temporal dynamics in viral shedding and transmissibility of COVID-19. Nature medicine. 2020;26(5):672-5.

20. Wölfel R, Corman VM, Guggemos W, Seilmaier M, Zange S, Müller MA, et al. Virological assessment of hospitalized patients with COVID-2019. Nature. 2020;581(7809):465-9.

21. Liu Y, Yan L-M, Wan L, Xiang T-X, Le A, Liu J-M, et al. Viral dynamics in mild and severe cases of COVID-19. The Lancet Infectious Diseases. 2020.

22. Wu Z, McGoogan JM. Characteristics of and important lessons from the coronavirus disease 2019 (COVID-19) outbreak in China: summary of a report of 72314 cases from the Chinese Center for Disease Control and Prevention. Jama. 2020;323(13):1239-42.

23. Organization WH. Laboratory testing for coronavirus disease 2019 (COVID-19) in suspected human cases: interim guidance, 2 March 2020. World Health Organization, 2020.

24. Radpour A, Bahrami-Motlagh H, Taaghi MT, Sedaghat A, Karimi MA, Hekmatnia A, et al. COVID-19 evaluation by low-dose High Resolution CT scans protocol. Academic Radiology. 2020.

25. Burki T. Outbreak of coronavirus disease. Lancet Infect Dis. 2019.

26. Guan W-j, Ni Z-y, Hu Y, Liang W-h, Ou C-q, He J-x, et al. Clinical characteristics of coronavirus disease 2019 in China. New England journal of medicine. 2020;382(18):1708-20.

27. Fang Y, Zhang H, Xie J, Lin M, Ying L, Pang P, et al. Sensitivity of chest CT for COVID-19: comparison to RT-PCR. Radiology. 2020:200432.

28. Shi H, Han X, Jiang N, Cao Y, Alwalid O, Gu J, et al. Radiological findings from 81 patients with COVID-19 pneumonia in Wuhan, China: a descriptive study. The Lancet Infectious Diseases. 2020.

29. Huang P, Liu T, Huang L, Liu H, Lei M, Xu W, et al. Use of chest CT in combination with negative RT-PCR assay for the 2019 novel coronavirus but high clinical suspicion. Radiology. 2020;295(1):22-3.

30. Xie X, Zhong Z, Zhao W, Zheng C, Wang F, Liu J. Chest CT for typical 2019-nCoV pneumonia: relationship to negative RT-PCR testing. Radiology. 2020:200343.

31. Nolan T, Hands RE, Bustin SA. Quantification of mRNA using real-time RT-PCR. Nature protocols. 2006;1(3):1559.

32. Yang Y, Yang M, Shen C, Wang F, Yuan J, Li J, et al. Evaluating the accuracy of different respiratory specimens in the laboratory diagnosis and monitoring the viral shedding of 2019-nCoV infections. MedRxiv 2020: 2020.02. 11.20021493. Sólo en caso de urgencia y si no se cuenta con otro donante podrá ser recolectado evaluando cuidadosamente los riesgos y beneficios Podría volver a ser elegido si no tuvo historia de infección respiratoria severa, hayan trascurrido.28.

33. Zhang Jj, Cao Yy, Dong X, Wang Bc, Liao My, Lin J, et al. Distinct characteristics of COVID-19 patients with initial rRT-PCR-positive and rRT-PCR-negative results for SARS-CoV-2. Allergy. 2020.

\section{List of Abbreviations:}

COVID-19: coronavirus disease 2019

SARS-CoV-2: Severe acute respiratory syndrom coronavirus2

rRT-PCR: Reverse transcription polymerase chain reaction

DNA: Deoxyribonucleic acid

RNA: Ribonucleic acid 
ARDS: acute respiratory distress syndrome

CT: Computed tomographic

WHO: World Health Organization

HRCT: high resolution computed tomography

IQR: Interquartile range

\section{Hosted file}

\selectlanguage\{english\}jdwl \selectlanguage\{english\}Vital sign.docx 3 pcr.docx available at https://authorea.com/users/336442/articles/462124-clinical-and-laboratory-findings-incovid-19-patients-comparison-of-rt-pcr-positive-and-negative-cases

\section{Hosted file}

PCR myngyn w mynh dr prmtrh. \selectlanguage\{english\}docx 2.docx available at https: //authorea.com/users/336442/articles/462124-clinical-and-laboratory-findings-in-covid19-patients-comparison-of-rt-pcr-positive-and-negative-cases 